\title{
Investigating the Potential Role of Visualisation in Natural Resource Decision-making
}

\author{
B. Otinpong ${ }^{1}$, S. Charters ${ }^{2}$, A. McKinnon² and B. Gidlow ${ }^{3}$ \\ ${ }^{1}$ Department of Computing, Ara Institute of Canterbury; PO Box 540, Christchurch 8140, New Zealand; \\ ${ }^{2}$ Department of Informatics and Enabling Technologies, ${ }^{3}$ Department of Social Science, Parks, Recreation, \\ Tourism and Sport; Lincoln University; PO Box 85084, Lincoln 7647, New Zealand
}

Email: bernard.otinpong@ara.ac.nz

\begin{abstract}
Computer-aided visualisation can be applied to natural environments to understand the impact of proposed developments or management strategies, but little evaluation of the effectiveness of these tools has been undertaken. In seeking to manage natural environments, it is desirable to model and understand these complex interactions in order to compare the outcomes of applying different management strategies.
\end{abstract}

The purpose of this study was to investigate whether there are significant differences in knowledge outcomes depending on the form in which visualisation of environmental changes is presented, using a case study of Te Waihora/Lake Ellesmere, a broad, shallow lagoon in the South Island of New Zealand.

Te Waihora/Lake Ellesmere is separated from the Pacific Ocean by the long narrow sandy Kaitorete Spit. Its unique position allows for it to be opened to the sea periodically to provide drainage and prevent flooding of surrounding farmlands. There is a lack of agreement among the diverse stakeholders regarding the appropriate levels at which the lake level should be maintained throughout the year.

We describe an interactive visualisation tool (ElleVis) which shows the effects of different water levels on the flora and fauna, as well as plants and animals living in and around the Lake. The tool allows users to input different opening scenarios and visualise the resulting impact on water levels around the lake at various times. It incorporates historical rainfall data from New Zealand's National Institute of Water and Atmospheric Research to deliver a graphical map display, including a summary table with a 'traffic light' status for lake values - birds, fish, farming and other stakeholder interests at different locations around the lake. The interactive nature of the ElleVis tool allows the stakeholders to compare Te Waihora/Lake Ellesmere under different opening scenarios using one interactive tool. However, it is possible, for example, that providing information about changes in lake behaviour in a carefully and clearly presented non-interactive form may be as successful as providing it in an interactive form of ElleVis.

In order to test for the effect of interactive versus non-interactive forms of visualization, we conducted an experiment with forty participants (randomly assigned to two test groups) who have various interests at Te Waihora. We provided them with either an interactive or a non-interactive form of visualisation. Results were recorded from a structured interview after the test. The findings revealed that interactive visualisation was key to advantageous learning about changes in environmental behaviour. We argue that the techniques presented have the potential to stimulate meaningful discussions in natural resource situations that involve contested resources or a multiplicity of interests, but at the same time, there is an urgent need for evaluation of such tools in participatory decision-making processes.

Keywords: Personal understanding, interactive visualisation, static visualisation, evaluation, visual simulation 


\section{INTRODUCTION}

Changes to the natural environments are often met with fierce resistance from stakeholders, some of which is because stakeholders fail to get an overall picture on how changes to their landscape will affect them. In order to overcome the initial resistance, projects often involve community consultation or engagement. The principle of community engagement holds that those who are affected by a decision have a right to be involved in the decisionmaking process. However, challenges exist in communicating these changes and management options to stakeholders and it has been suggested that visualisation techniques could facilitate stakeholder engagement, increase understanding and improve the quality of decision making (Orland, Budthimedhee, \& Uusitalo, 2001; Sheppard, 2005).

Visualisations can be applied to natural environments to understand the impact of proposed developments or proposed management strategies. Visualisations can be interactive or non-interactive, with each having their advantages depending on the purpose. For example, authors such as Tufte and Graves-Morris (1983) argue that non-interactive visualisations are helpful when planners want to inform decision-makers about relatively simple and straightforward issues. Interactive visualisation has important implications for decision-making in different contexts. Interactivity enhances the restructuring of information and the selection of meaningful information. The way people perceive and interact with visualisations can influence their understanding of issues and the usefulness of the visualisation systems (Zudilova-Seinstra, Adriaansen, \& van Liere, 2009). It is on this basis that an interactive visualisation tool (ElleVis) together in its non-interactive form was developed and presented as a medium to communicate to Te Waihora/Lake Ellesmere stakeholders with diverse opinions/interest. This is used to address the question of whether interactive visualisation provides greater knowledge gains than a noninteractive form of information provision. The research question we are exploring is:

“Does use of an interactive visualisation make a significantly greater contribution to stakeholder's personal understanding of the impacts of lake opening regimes on lake values at Te Waihora/Lake Ellesmere than use of a non-interactive visualisation?"

For the purposes of this study, the term "lake values" refers to farming, nature conservation and other stakeholder interests, which are affected by the different opening regimes at Te Waihora/Lake Ellesmere. These values have been identified by the several stakeholders as being of significance to them (Hearnshaw \& Hughey, 2010).

\section{STUDY SITE SELECTION}

Te Waihora/Lake Ellesmere in the Canterbury region of the South Island of New Zealand is a broad, shallow lagoon separated from the Pacific Ocean by the long, narrow, sandy Kaitorete Spit. Its unique position allows it to be mechanically "opened" to the sea periodically in order to provide drainage and prevent flooding of surrounding farmlands. There is a lack of agreement between the stakeholders as to the levels at which the lake should be maintained and how that level should be manipulated. Different lake levels are perceived to advantage some stakeholder's interests, such as farming, fishes, and birds, and to disadvantage others with recreational and cultural interests.

\section{ELLeVIS}

To determine whether there are significant differences in knowledge acquisition depending on the form in which visualisation of environmental changes is presented, an interactive visualisation tool (ElleVis) was developed. The tool is designed to provide information which a range of stakeholders would need to aid their decision making about the lake. The primary source of stakeholder requirements was the lake opening consent (CRC 042860) from the local Regional Council (Environment Canterbury). This identified the need for the visualisation to show:

- $\quad$ The level and area of the lake at different lake levels

- The shoreline and farmlands covered at different lake levels

- The impact on birdlife and native fisheries at different lake levels

- The impact on livestock along the surrounding farmlands at different lake levels

The design of ElleVis shown below in Figure 1 has three main components: a "traffic light" summary table showing overview information on lake values; a map showing lake extent; and a lake level time series graph. The tool allows users to configure lake opening scenarios and select the conditions under which they are viewed. The data for the visualisation are generated by the Plover model (Raffensperger, 2009) which simulates the behaviour of the lake. The Plover model, which has been peer reviewed by several scientists consists of 38 years of real 
hydrological data on the water inflows and outflows of Te Waihora/Lake Ellesmere, lake margins, lake area and lake volumes.

The time series graph shown in Figure 1 shows the level above mean sea level (amsl) of the lake over the year. The graph is augmented with blue shaded areas that show when the lake has been opened to the sea. The shading gives a visual indication of the frequency and duration of lake openings.

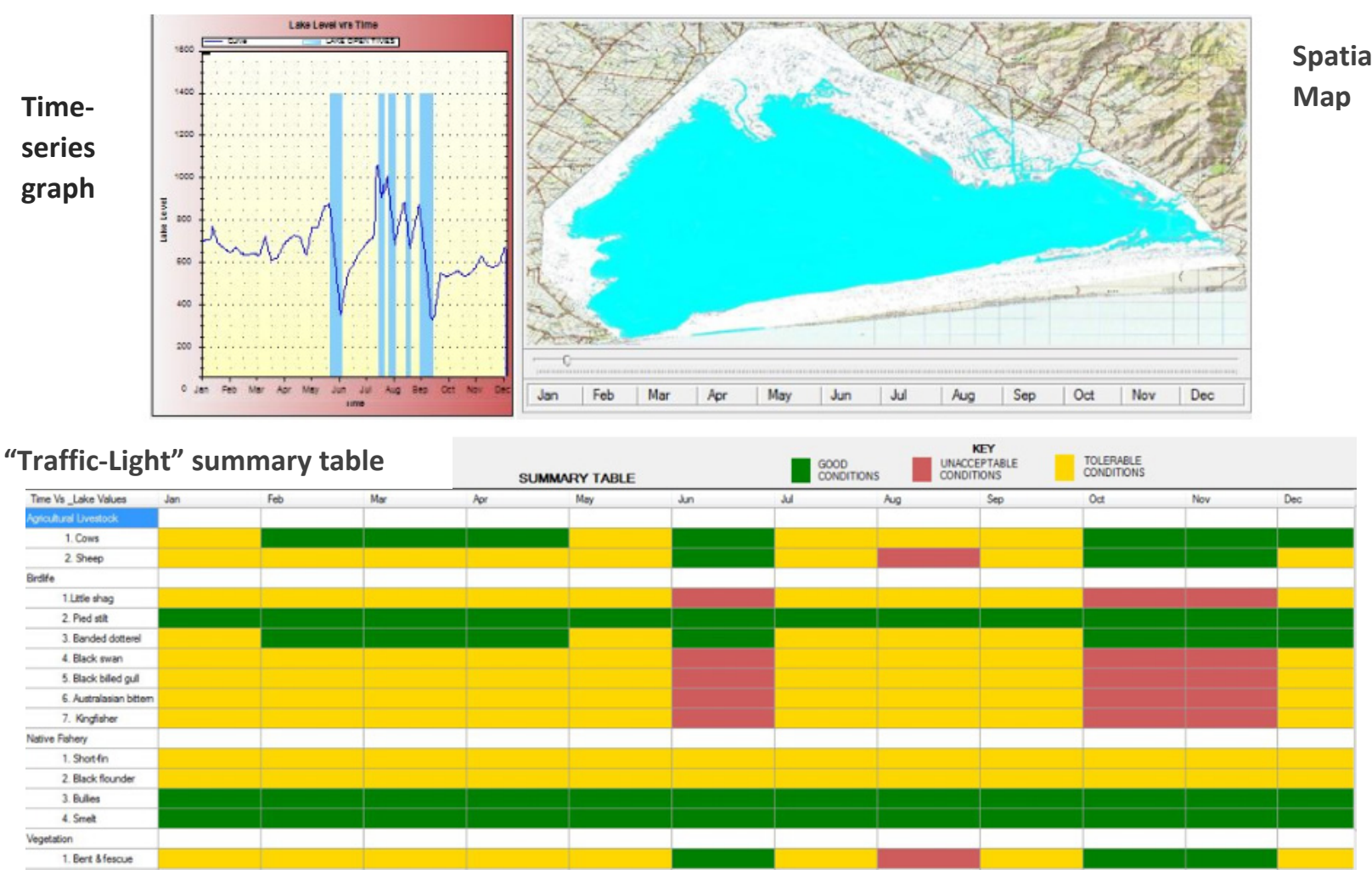

Figure 1. ElleVis visualisation interface

The "traffic light" summary table shown in Figure 1 represents an overview of information on the impact of the opening regimes on lake values. The cells are colour coded to help participants to distinguish which lake values are good (green), tolerable (amber) and unacceptable (red). Each row in the table represents one lake value (for example "sheep", "banded dotterels") with each colour in a cell representing the lake level conditions for that month. Each cell in the summary table contains a computed average of lake levels for a month based on the opening regime entered by the user. The summary table view provides stakeholders with overview information on lake values at a glance for the opening regime entered.

The interactive map shown in Figure 1 allows stakeholders to see the consequences of different opening regimes on the extent of the lake. The image is a topographic map with the lake superimposed. A slider beneath the image allows the extent of the lake during the year to be explored on a day by day basis. A non-interactive visualisation consisting of screen shots taken from the interactive tool (ElleVis) but presented in paper form was also developed as part of a medium to communicate to participants of the study.

\section{STUDY DESIGN}

A split-sample pre-test and post-test design was used for this study (Dimitrov \& Rumrill, 2003). Using this design, participants are pretested, exposed to the intervention (Interactive and Non-interactive - paper based - forms of ElleVis), and then post-tested.

Figure 2 shows an overview of the process involved in measuring change in participants' understanding using different forms of information provision. One group - Group A - experienced the interactive visualisation while the other - Group B - experienced screen shots taken from ElleVis as a non-interactive/paper-based visualisation. 
For the purposes of this study, the target population was people who have an interest and understanding in Te Waihora/Lake Ellesmere and its future management, rather than the general public; for example, local residents, farmers, fishers and recreationists around the lake and employees of governmental and nongovernmental organisations who have vested interests in the lake. It was anticipated that their interest in the lake would encourage such people to participate. Forty participants (20 in each group, A and B) were used for the study.

Letters for participation in the study were sent to all participants. The study was conducted individually at a time and place most convenient to each participant.

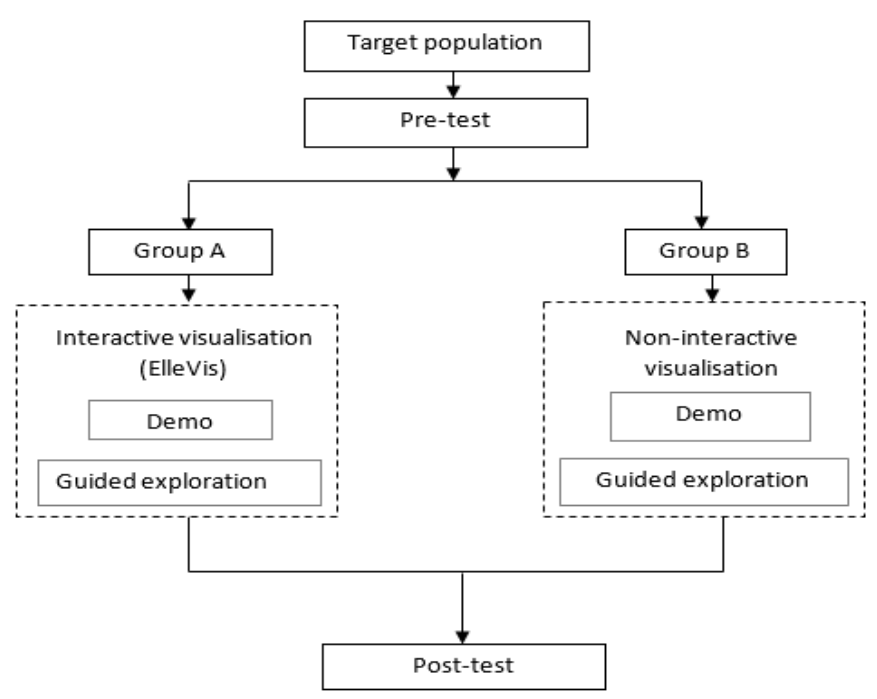

Figure 2. Overview of study design

\subsection{Conducting the study}

Participants were randomly assigned to two treatment groups - Group A and Group B. Group A participated in the interactive version of ElleVis, Group B the non-interactive version. The same pre-test and post-test was done on all participants.

After the pre-test, Group A participants were exposed to a visual demonstration intended to show them how to use the visualisation tool, which is customised software. The demonstration involved participants 'seeing and interacting' with the visualisation but not exploring all possible scenarios. Then they were given guidedexploration tasks using the interactive visualisation tool, ElleVis. The purpose of the guided exploration is for participants to be informed of lake values that they may be less familiar with under different opening regimes. Finally, the participants were post-tested.

Group B participants, after the pre-test were shown how to locate and interpret the different features of ElleVis using the paper-based visualisation. They were then given the same guided-exploration tasks as Group A, however this group completed them using the non-interactive visualisation rather than the interactive visualisation. A posttest then took place.

\subsection{Data Analysis}

A series of questions was developed to gather feedback on the issues participants encountered using the visualisations. The following prompts embedded in questions were used to guide the discussion: usefulness of the visualisation (non-interactive or interactive); most effective component of the visualisation; alternate forms of information provision to assist in understanding of lake issues; usability of the visualisation tool (non-interactive or interactive); accuracy of the visualisation in depicting the lake behaviour; increase in knowledge about the lake after using the visualisation; benefits of the visualisation (non-interactive or interactive); other general comments about the way information was presented. The interviews were transcribed verbatim and the data organised into groups which relate to areas of interest as generated by the 'prompts' above.

\section{RESULTS}

Data was arranged using the NVivo software based on 'self-emerging' themes from the transcripts (Halcomb \& Davidson, 2006). These themes were examined in relation to the key research question. Themes explored include: discussions highlighting the interactivity of the visualisations; effectiveness of the visualisation format; usefulness of the visualisation; ease of use of the visualisation; the perceived benefits and drawbacks associated with the form or forms of information presented.

All participants were unanimous in their response that the visualisations were useful in learning about features of the lake and that of the plants and animals in and around it. A majority of the participants (30), which represents an even split between Groups A and B, indicated that the "summary table" is the most effective component of the 
visualisation. Five Group A (compared with two Group B) participants found the spatial image to be the most effective component of the visualisation. Key quotes are provided to illustrate results and combined with discussion in the subsections below.

\subsection{Interactive visualisation techniques provide added communication value}

All Group A participants who used the interactive visualisation became increasingly engaged ${ }^{1}$ when using the tool, and found the possibilities the tool offers to be helpful. The format of the visualisation increased their knowledge in several ways to an extent that they conveyed the excitement that the tool offers by thinking how the tool might be used in future. For example, one participant from Group A remarked:

"I like the layout of the visualisation. In fact I am very happy with the way they are arranged on the screen. It gives me a good visual of what is happening down there, and this has enhanced my understanding in many ways"

Another Group A participant noted:

"This is a great visual. The components of the visualisation are just great. At least it gives you a sense of factors at play with each other"

Responses from the participants suggest that the interactivity of ElleVis could be used in other environmental management contexts, more preferably it can be incorporated into decision-making. The interactivity of ElleVis was also perceived to be very valuable as a tool in clarifying contentious issues, and thus could be applied to other decision-making or public participatory processes in environmental management situations where conflicts arise. In fact, one Group A participant noted that:

"I think the visualisations are really good and has improved my overall understanding of the lake...I see the possibility of using this format for different situations. This works for a lot of environment situations, but also in other situations where you use collaborative decisions for decision making. So I see this as a possibility of using this programmable interface in other situations as well".

Interactivity seems to simulate discussions with participants, and it also seems easier for the participants to express other situations the tool may be used for. However, participants who used the non-interactive ElleVis were less engaged in providing alternatives to the use of the visualisation. This corroborates other works from authors such as Orland et al. (2001) that interactivity increases participants' engagement.

\subsection{Interactive nature of spatial image improves understanding}

In response to the question, "Which of the components of the visualisation tool did you find most effective in increasing your understanding about lake management issues at Te Waihora?" five Group A (compared with two Group B) participants, found the spatial map to be the most effective component of the visualisation. Participants using the interactive ElleVis were able to explore the features of the spatial map to see how different opening regimes impacted upon land inundation on the surrounding farmland, which was not possible for Group B participants because of the static nature of the visualisations. Accompanying comments from Group A participants include:

"The spatial map is the most effective component because of its interactive nature. With the spatial map you can see the street, the lands that will be covered by water.... This increased my knowledge about how the lake interacts with the land and its environs. Surely, this will benefit those of us here to know when it will be like that. If you have been here for a long time you will know what's going on...."

Another participant from Group A remarked:

"I like the spatial map because it is very dynamic. I particularly like the interaction between the water levels and the surrounding lands. This has increased my understanding in several ways on how rise in water levels of the lake could impact the land"

For the two Group B participants who found the spatial map helpful, one remarked:

\footnotetext{
${ }^{1}$ Measurement of engagement involved the excitement participants conveyed in using the tool by readily providing other situations on how ElleVis might be used in future. This data was recorded using handwritten notes.
} 
"I like the idea of the spatial map and the way it works for the presentation. If only I could see how it works in reality. It will help me immensely to know when these surrounding lands will flood in different seasons"

The other Group B participant noted:

"I think the concept of the spatial map is rather helpful .... This has helped me appreciate this tool as it has enhanced my understanding in several ways. Only if I could have a play at it on a computer screen and not just outputs like these will help me explore some scenarios more to be better informed on when high water levels kind of flood the beaches"

The above quotes from these Group A and B participants suggests that the spatial map was more effective when in an 'interactive form' rather than screen shots form (see Otinpong et al., 2015). The interactivity of ElleVis provided Group A participants with an improved level of insight as they were able to make use of information that would otherwise be impossible to recognise. For example, subtle changes in opening regimes can have a severe impact on the surrounding farmlands, but this is hardly noticeable when using non-interactive visualisations. The interactivity of ElleVis makes information more easily evaluated and compared than noninteractive visualisation. The results of this research match those of earlier studies (Bateman, Day, Jones, \& Jude, 2009; Lowe \& Schnotz, 2008; Tversky, Morrison, \& Betrancourt, 2002) which suggest that interactivity can enhance comprehension and learning by stimulating cognitive processing (Card, Mackinlay, \& Shneiderman, 1999).

\subsection{Discussion highlighting the interactivity of ElleVis}

The results and discussion in Sections 5.1 and 5.2 strongly suggest that the use of interactivity may have afforded Group A participants more control over information, and increased their ability to manipulate and use information in performing tasks, thus increasing their understanding more than Group B participants who used the noninteractive ElleVis. The data presented in the above sections, however, made it clear that both groups - A and B displayed increased learning, which means that 'interactivity' alone could not account for all such increases (Otinpong et al., 2015 covers these issues in greater depth). The next section therefore elaborates on the coverage in that section, discussing similarities in learning experienced by both groups.

\subsection{Similarities between Groups A and B}

One of the key advantages of the visualisation highlighted by the responses from both Groups A and B was the use of colours (traffic lights) to display the impact of lake level conditions for animals and plants in and around the lake. Both the interactivity and the screen shots made use of colour. In particular, the participants felt that the way the visualisation interface was presented may offer a means of dialogue, or "common currency" as Orland et al., (2001) have described it, between the conflicting parties of Te Waihora/Lake Ellesmere by helping them to understand the impact associated with different opening regimes on lake values.

Comments from both groups of participants indicated that the visualisations were an improvement of the traditional formats such as leaflets and direct mail-outs that participants were accustomed to receiving/seeing when gaining information about Te Waihora/Lake Ellesmere. For example, a participant from Group B noted: "These presentations are an improvement on the leaflets they use to drop in our mailboxes explaining the management options of Te Waihora. These presentations has enhanced my understanding on how the lake operates".

For some Group B participants, even the screen shots were a 'new' way of presenting information about the Lake and thus had a 'Wow' factor. These results confirm other studies that have investigated the potential role and value of visualisation techniques in environmental decision-making by (Appleton \& Lovett, 2003; Bateman et al., 2009; Brown et al., 2006; Dockerty et al., 2006).

However, the authors acknowledge that further research is required to shed some light on how improvement in participants' understanding might have occurred through interactivity.

\section{CONCLUSION AND FUTURE RESEARCH}

We gained important insights from this study. It established that; (a) the use of both interactive and non-interactive visualisation improved knowledge and; (b) interactive visualisation was the more successful form of visualisation than the non-interactive visualisation in improving knowledge. 
The design and implementation of ElleVis offers the participants' a learning environment that enhances their understanding, which suggests that those designing decision support systems and conducting visualisation research that involve contested resources are well-advised to employ a suite of visualisation formats. Future work could also look at applying the visualisation format of ElleVis to other environmental situations such as other bodies of water, the siting of irrigation-systems, the creation of new dams and the removal of existing dams. Further refinements could be made to enhance some of the features of ElleVis so that it provides users with a better understanding of the uncertainties and risks associated with decision-making. This would provide evidence of the robustness of the approach taken in ElleVis when taken beyond the specific Te Waihora/Lake Ellesmere context.

Given that no measures of motivation were conducted during the studies, future research could incorporate such measurements to see if these contribute to variations in participants' performance and/or engagement. Issues of motivation are important because they have been found to increase levels of understanding and productivity amongst participants (Davis, Bagozzi, \& Warshaw, 1992).

\section{REFERENCES}

Bateman, I. J., Day, B. H., Jones, A. P., \& Jude, S. (2009). Reducing gain-loss asymmetry: a virtual reality choice experiment valuing land use change. Journal of Environmental Economics and Management, 58(1), 106-118.

Card, S. K., Mackinlay, J. D., \& Shneiderman, B. (1999). Readings in information visualization: using vision to think. San Francisco, CA, USA: Morgan Kaufmann Inc.

Davis, F. D., Bagozzi, R. P., \& Warshaw, P. R. (1992). Extrinsic and intrinsic motivation to use computers in the workplace1. Journal of applied social psychology, 22(14), 1111-1132.

Dimitrov, D. M., \& Rumrill, P. D. (2003). Pretest-posttest designs and measurement of change. Work: A Journal of Prevention, Assessment and Rehabilitation, 20(2), 159-165.

Halcomb, E. J., \& Davidson, P. M. (2006). Is verbatim transcription of interview data always necessary? Applied Nursing Research, 19(1), 38-42.

Hearnshaw, E. J. S., \& Hughey, K. F. D. (2010). A tolerance range approach for the investigation of values provided by Te Waihora/Lake Ellesmere Land Environment and People Research Report (pp. 67). Christchurch: Centre for Land, Environment \& People.

Kristensen, T., \& Gabrielsen, G. (2000). The Meaning of Colours in Design Research Paper no 2. Copenhagen: Copenhagen Business School, Department of Marketing.

Lorie, M. (2006). Informed Consent through Collaborative Modeling and Transparent Decision-Making: The Lake Ontario St. Lawrence River Study. Paper presented at the World Environmental and Water Resource Congress 2006:Examining the Confluence of Environmental and Water Concerns, 21-25 May, Omaha,Nebraska:USA.

Orland, B., Budthimedhee, K., \& Uusitalo, J. (2001). Considering virtual worlds as representations of landscape realities and as tools for landscape planning. Landscape and Urban Planning, 54(1-4), 139-148.

Otinpong, B., Charters, S., McKinnon, A., \& Gidlow, B. (2015). A comparison of results between interactive and non-interactive forms of visualisation to improve learning. A case study of Te Waihora/Lake Ellesmere, Canterbury, New Zealand. . 21st International Congress on Modelling and Simulation(MODSIM2015). Modelling and Simulation Society of Australia and New Zealand, pp. 1916-1922.

Raffensperger, J. F. (2009). Introducing PLOVER 2k: Planning openings and Values for Lake Ellesmere's Resilience. Christchurch.

Sheppard, S. R. J. (2005). Landscape visualisation and climate change: the potential for influencing perceptions and behaviour. Environmental Science \& Policy, 8(6), 637-654.

Tversky, B., Morrison, J. B., \& Betrancourt, M. (2002). Animation: can it facilitate? International journal of human-computer studies, 57(4), 247-262.

Zudilova-Seinstra, E. V., Adriaansen, T., \& van Liere, R. (2009). Overview of Interactive Visualization Trends in Interactive Visualization, State-of-the-Art Survey, in series Advanced Information and Knowledge Processing (pp. 3-15). London: Springer. 